\title{
Binding of CLL Subset 4 B Cell Receptor Immunoglobulins to Viable Human Memory B Lymphocytes Requires a Distinctive IGKV Somatic Mutation
}

\author{
Rosa Catera, ${ }^{1}$ Yun Liu, ${ }^{1,2}$ Chao Gao, ${ }^{3}$ Xiao-Jie Yan, ${ }^{1}$ Amanda Magli, ${ }^{1}$ Steven L Allen, ${ }^{1,2,4}$ Jonathan E Kolitz, ${ }^{1,2,4}$ \\ Kanti R Rai, ${ }^{1,2,4}$ Charles C Chu, ${ }^{1,2,4}$ Ten Feizi, ${ }^{3}$ Kostas Stamatopoulos, ${ }^{5}$ and Nicholas Chiorazzi $i^{1,2,4}$ \\ ${ }^{1}$ Karches Center for Oncology Research, The Feinstein Institute for Medical Research, Manhasset, New York, United States of \\ America; ${ }^{2}$ Hofstra Northwell School of Medicine, Hempstead, New York, United States of America; ${ }^{3}$ Glycosciences Laboratory, \\ Department of Medicine, Imperial College London, London, United Kingdom; ${ }^{4}$ Department of Medicine, Hofstra Northwell School \\ of Medicine, Hempstead, New York, United States of America; and ${ }^{5}$ Institute of Applied Biosciences, Centre for Research and \\ Technology-Hellas, Thessaloniki, Greece
}

\begin{abstract}
Amino acid replacement mutations in certain chronic lymphocytic leukemia (CLL) stereotyped B cell receptor (BCR) immunoglobulins (IGs) at defined positions within antigen-binding sites strongly imply antigen selection. Prime examples of this are CLL subset 4 BCR IGs using IGHV4-34/IGHD5-18/IGHJ6 and IGKV2-30/IGKJ2 rearrangements. Conspicuously, and unlike most CLL IGs, subset 4 IGs do not bind apoptotic cells. By testing the (auto)antigenic reactivities of subset 4 IGs toward viable lymphoid-lineage cells and specific autoantigens typically bound by IGHV4-34+ IGs, we found that IGs from both subset 4 and non-subset 4 IGHV434-expressing CLL cases bound naïve B cells. However, only subset 4 IGs reacted with memory B cells. Furthermore, subset 4 IGs did not bind DNA nor i or I carbohydrate antigens that are common targets of IGHV4-34-utilizing antibodies in systemic lupus erythematosus and cold agglutinin disease, respectively. Notably, we found that subset 4 IG binding to memory B lymphocytes depends on an aspartic acid at position 66 of FR3 in the rearranged IGKV2-30 gene; this amino acid residue is acquired by somatic mutation. Our findings illustrate the importance of positive and negative selection criteria for structural elements in CLL IGs and suggest that autoantigens driving normal B cells to become subset 4 CLL cells differ from those driving IGHV4-34 $\mathrm{B}$ cells in other diseases.
\end{abstract}

Online address: http://www.molmed.org

doi: $10.2119 / \mathrm{molmed} .2017 .00003$

\section{INTRODUCTION}

Considerable evidence suggests that normal B cells ushered into the leukemic state in chronic lymphocytic leukemia (CLL) are selected by the structure of their surface membrane immunoglobulin (IG) antigen-binding site (1-3). Probably the most dramatic evidence for this is that about one-third of CLL cases exhibit "stereotyped" B cell receptor (BCR) IGs, characterized by very similar VH CDR3 amino acid sequences, usually encoded by specific combinations of IGHV-IG$H D-I G H J$ genes. The concept of stereotyped BCR IGs in CLL has evolved from a scientific enigma (4-6) to a defined entity $(7,8)$ to a paradigmatic principle $(9-11)$ that supports the concept that leukemic B cell clones are selected for transformation based on structural restrictions in their antigen-binding domains $(1,12,13)$.

This principle has been further elaborated in certain CLL stereotyped subsets for which specific amino acid replace-

Address correspondence to Nicholas Chiorazzi, The Feinstein Institute for Medical Research, Experimental Immunology, 350 Community Drive, Manhasset, NY 11030 , USA. Phone: 516-562-1090; Fax: 516-562-1011; E-mail: NChizzi@NSHS.edu

Submitted January 3, 2017; Accepted for Publication January 5, 2017; Published Online (www.molmed.org) January 12, 2017.

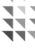

Feinstein Institute for Medical Research Northwell Health"

ment mutations have been found at defined positions within the leukemic B cell's antigen-binding site (14). A prime example of this is subset 4 , a group of patients originally defined by the use of IGHV4-34/IGHD5-18/IGHJ6 and IGKV230/IGKJ2 rearrangements $(7,9)$. The CLL clones in this group are always IgG isotype switched and always exhibit IG somatic mutations (IGHV-mutated CLL, M-CLL). Of note, the IG mutations in the clones of many subset 4 patients are shared, focusing on specific positions in the $\mathrm{H}$ and $\mathrm{L}$ chain rearrangements and often introducing the same or chemically similar amino acids at those sites (14). Finally, subset 4 BCR IGs can continue to acquire somatic mutations over time $(15,16)$, implying that antigen selection operates during clonal evolution.

Clinically, patients in subset 4 follow indolent clinical courses, faring even better than patients with mutated IGHV 
genes (16). This is surprising, because subset 4 patients often develop the disease at an earlier age than other patients who fall into other stereotyped subsets or with CLL in general (16). One possible explanation for their long and relatively benign clinical courses is the frequent inability to signal through the BCR $(17,18)$, since in other patients such signaling can correlate with poor survival (19).

We $(20,21)$ and others (22) have shown that the mAbs expressed by the leukemic B cells of $\sim 50 \%$ of CLL patients bind molecules on the surface of apoptotic human $\mathrm{T}$ and $\mathrm{B}$ lymphocytes. It is likely that such reactivity is directed toward antigens that are usually intracellular and become accessible on the surface membrane during apoptosis $(20,21)$. However, a distinguishing feature of subset 4 IGs mAbs is their inability to react with apoptotic cells $(20,21)$.

In this study, we evaluated the (auto) antigenic specificities of subset 4 , using three mAbs belonging to the subset. Specifically, we evaluated the ability of these mAbs to bind viable human lymphoid cells as well as other autoantigenic targets that are often bound by mAbs using the IGHV4-34 gene. By comparing these results with appropriate control mAbs, we determined that subset $4 \mathrm{mAbs}$ bind viable human naïve and memory B cells. We also found that, remarkably, binding to memory B cells is dependent on the acquisition, via somatic mutation, of a single amino acid at a defined position in the rearranged IGKV2-30 gene. Furthermore, we determined a surprising loss of subset 4 antibody reactivity with two autoantigens commonly bound by IGHV4-34-expressing mAbs, namely DNA and the I/i carbohydrate antigens. Taken together, these findings suggest that the autoantigens driving the clonal evolution of normal B cells to become subset 4 CLL cells are distinct from those usually associated with IGHV4-34 IG binding in other disease settings. In the broader picture, this detailed characterization of antigenic specificity of subset 4, a prototype for clinically indolent, good prognosis CLL, advances our understanding of how positive and negative selection for specific structural elements in CLL BCRs shape clonal behavior and ultimately patient outcome.

\section{MATERIALS AND METHODS}

\section{Cloning, Expression and Purification of CLL mAbs}

RNA from blood mononuclear cells of CLL patients was extracted and converted into cDNA as previously described (12). Cloning, expression and purification of mAbs were performed as reported (23).

\section{Site-Directed Mutagenesis}

Targeted mutagenesis of the IGHV$I G H D-I G H J$ and IGKV-IGKJ sequence of CLL mAbs was performed by GENEWIZ.

\section{Cell Immunofluorescence and Flow Cytometric Analysis}

Apoptosis in cell lines was induced by $\gamma$-irradiation (4000-5000 R) for $\sim 15 \mathrm{~h}$ before staining. Cells were then incubated with CLL mAbs $(5-50 \mu \mathrm{g} / \mathrm{mL})$ for $1 \mathrm{~h}$ at $4^{\circ} \mathrm{C}$ and binding detected by FITC-conjugated $\mathrm{F}\left(\mathrm{ab}^{\prime}\right)_{2}$ goat anti-human IgG (Southern Biotech), or by streptavidin PE-Cy7 (eBioscience) when biotinconjugated mAbs (sulfo-NHS-LC-biotin, Thermo Scientific) were used. Apoptosis was measured by staining with annexin V-PE (BD Pharmingen) as recommended by the vendor.

Cells isolated from tonsil were stained using exclusively biotin-conjugated CLL-derived mAbs. To distinguish different lymphocyte populations in tonsil samples, the following Abs were used: Pacific Blue mouse anti-human IgM (BioLegend, clone MHM-88), FITC goat $\mathrm{F}(\mathrm{ab})_{2}$ anti-human IgD (Southern Biotech), Alexa Fluor 700 mouse antihuman CD38 (eBioscience, clone HIT2), and AmCyan mouse anti-human CD3 (clone SK7), PerCP mouse anti-human CD19 (clone 4G7), APC mouse antihuman CD27 (clone L128) and PE mouse anti-human CD24 (clone ML5) (all BD Biosciences). Samples were acquired using an LSRII flow cytometer (Becton
Dickinson) and analyzed using FlowJo (LiveTree) software.

\section{mAb Binding to DNA by Enzyme- Linked Immunosorbent Assay}

To determine $\mathrm{mAb}$ binding to doubleand-single stranded DNA, QUANTA Lite $^{\text {TM }}$ dsDNA and QUANTA Lite ${ }^{\text {TM }}$ ssDNA enzyme-linked immunosorbent assay (ELISA) kits (INOVA Diagnostics) were used. mAbs were tested following the manufacturer's instructions at $25 \mathrm{ug} / \mathrm{mL}$. The signal-to-unit conversion was calculated and positive cutoff values were determined according to the manufacturer's instruction.

\section{Lipid-Linked Oligosaccharide Probes}

Two hexasaccharides, pLNH and pLNnH, from Dextra and an octasaccharide, $\mathrm{LNnO}$, from Elicityl were analyzed as lipid-linked glycan probes, neoglycolipids (NGLs) derived by conjugation to a phospholipid L-1,2-dihexadecyl-snglycero-3-phosphoethanolamine (DHPE) (24). A ceramide-linked octasaccharide I-octa was isolated from human red cells (gift of Peter Hanfland). The glycan sequences are given in Figure 3. These NGLs and glycolipids were part of a microarray of 79 NGL and glycolipid probes encompassing blood groups $\mathrm{A}, \mathrm{B}, \mathrm{H}, \mathrm{Le}^{\mathrm{a}}, \mathrm{Le}^{\mathrm{b}}, \mathrm{Le}^{\mathrm{x}}$ and $\mathrm{Le}^{\mathrm{y}}$ related sequences based on linear and branched backbones (collectively designated CLL array Set 1, which is described in detail elsewhere). These lipidlinked probes were robotically arrayed in duplicate at 2 and $5 \mathrm{fmol}$ per spot on nitrocellulose-coated glass slides using a noncontact instrument as described (25).

\section{Microarray Analyses for Anti-i- and Anti-I-type Reactivity}

Microarray binding analyses were performed at $4^{\circ} \mathrm{C}$ essentially as described (26). In brief, after blocking with $3 \% \mathrm{w} / \mathrm{v}$ bovine serum albumin (Sigma-Aldrich) in HEPES-buffered saline (5 mM HEPES, $\mathrm{pH}$ 7.4, $150 \mathrm{mM} \mathrm{NaCl}, 5 \mathrm{mM} \mathrm{CaCl}$ ), the microarrays were probed with the following CLL mAbs: 141, 282, 183, 240 and 342 , all at $10 \mu \mathrm{g} / \mathrm{mL}$. The five $\mathrm{mAbs}$ were used in their native configuration as 
well as precomplexed with anti-human IgG to render the native molecule multimeric; this resulted in increased avidity of binding to ligands and allowed comparisons to IgM molecules. Data obtained with precomplexed and native bivalent IGs were the same. As positive controls, human IgM anti-i and anti-I sera from the Feizi laboratory collection, designated anti-i P1A ELL (27) and anti-I Ma (27), respectively, were analyzed at 1:100 dilution; moreover, a mouse IgM culture supernatant designated Fc10.2 (28) was tested undiluted. Binding was detected using biotinylated anti-human IgG (Vector Laboratories, anti-H + L chain), or biotinylated anti-human IgM (Vector Laboratories, $\mu$-chain specific), or biotinylated anti-mouse IgM (Sigma-Aldridge, $\mu$-chain specific), all at 1:200 dilution, followed by Alexa Fluor-647-labeled streptavidin (Molecular Probes, Thermo-Fisher Scientific) at $1 \mu \mathrm{g} / \mathrm{mL}$. Imaging and data analysis were as described $(24,29)$. Binding signals for $\mathrm{pLNH}$, pLNnH and $\mathrm{LNnO}$ were probe-dose-dependent. Results shown are at $5 \mathrm{fmol} / \mathrm{spot}$.

\section{RESULTS}

CLL Subset 4 mAbs Bind Preferentially to Antigen(s) on the Surface of Viable Human B Lymphocytes

To understand the unexpected lack of binding of subset $4 \mathrm{mAbs}$ to apoptotic cells $(20,21)$, we tested three mAbs from CLL clones belonging to this stereotyped subset (mAbs 183, 240 and 342) for reactivity with viable and apoptotic human lymphoid cells. These results were compared with those obtained using two different CLL clones whose mAbs either use the same IGHV4-34 gene without a stereotyped rearrangement (mAb141), or use a different IGHV gene and belong to a different stereotyped subset (no. 1) with a distinct BCR IG encoded by IGHV1-3/ IGHD6-19/IGHJ4 and IGKV1(D)39/IGKJ2 gene rearrangements (mAb360).

$\gamma$-irradiated Ramos B and Jurkat $T$ cell line cells were exposed to the above mAbs under nonpermeabilizing conditions, and binding to the cell surface was detected by flow cytometry. Notably, each of the mAbs belonging to subset 4 (nos. 183, 240 and 342) bound to live $B$ cells and, to a much lesser degree, to live $\mathrm{T}$ cells (defined as annexin $\mathrm{V}^{-}$) compared to apoptotic/dead B and T cells (defined as annexin $\mathrm{V}^{+}$) (Figure 1A, three right panels). Meanwhile, as predicted from our previous studies $(20,21)$, the subset $1 \mathrm{mAb360}$ bound to apoptotic cells and minimally to viable cells (Figure 1A). In addition, mAb141, which uses IGHV434 but without a stereotyped rearrangement and therefore with a different $\mathrm{VH}$ CDR3 structure, showed low-level binding to annexin $\mathrm{V}^{-}$cells (Figure 1A and references 20,21). Figure 1B shows the binding of mAbs 183, 240 and 342 to live $\mathrm{B}$ and $\mathrm{T}$ cells (based on an average of four independent experiments).

These data indicate that subset 4 IGs display selective reactivity with viable human lymphocytes, with this reactivity being much greater for $\mathrm{B}$ than for T cells. Hence, the antigens recognized by subset $4 \mathrm{mAbs}$ are constitutively present on the surface of viable B cells, unlike those antigens brought to the cell surface membrane during apoptosis that are recognized by most other CLL mAbs.

\section{CLL Subset 4 mAbs Recognize Memory and Naïve Subpopulations of Tonsillar B Cells}

To verify that the above reactivity of subset 4 mAbs with viable human B cells was not limited to transformed Ramos B cells, we analyzed the binding characteristics of these mAbs with human B lymphocytes from tonsillectomy samples. CLL IGs bound well to CD19 ${ }^{+}$(Figure 2A, right column) but minimally, at best, to $\mathrm{CD}^{+}$(Figure 2A, left column) tonsil cells, in agreement with the findings above.

Intriguingly, the CLL subset $4 \mathrm{mAbs}$ distinguished two subpopulations of $\mathrm{CD}_{19}{ }^{+}$cells, one with low and another with higher $\mathrm{mAb}$ binding (Figure 2A). In contrast, the IGHV4-34 ${ }^{+}$non-subset 4 mAb141 recognized predominantly a single CD $19^{+}$population.

To further characterize the two tonsillar B cell subpopulations that the subset
4 IGs selectively bound to and determine which of the two subset 4-reactive populations were bound by the IGHV4-34 non-subset $4 \mathrm{mAb} 141$, we exposed the tonsil samples to a panel of mAbs and polyclonal Abs (pAbs) that distinguish naïve from memory human $B$ cells. Gating on the $\mathrm{CD} 19^{+} / \mathrm{CLL}$ subset $4 \mathrm{mAb} \mathrm{b}^{\text {bright }}$ population indicated that these cells were mostly $\mathrm{CD} 27^{-} / \mathrm{IgD}^{+} / \mathrm{CD} 38^{\text {low }}$ (Figure 2B), a phenotype compatible with that of naïve B cells. These cells also expressed surface membrane (sm) IgM (Figure 2B) and were CD24 $4^{+}$(data not shown). In contrast, the majority of the $\mathrm{CD}^{+} 9^{+} / \mathrm{CLL}$ subset $4 \mathrm{mAb}^{\text {dim }}$ cells exhibited a $\mathrm{CD} 27^{+} / \mathrm{IgD}^{-} / \mathrm{IgM}^{-/+} / \mathrm{CD} 38^{\text {high }}$ phenotype (Figure $2 \mathrm{~B}$ ), consistent with memory B cells. Finally, when analyzing the B-lymphocyte subpopulation bound by $\mathrm{mAb} 141$, it became clear that the IGHV4-34 $4^{+}$non-subset $4 \mathrm{mAb}$ bound the corresponding CD19 ${ }^{+} /$CLL subset $4 \mathrm{mAb}^{\text {bright }}$ naïve $\mathrm{B}$ cell population $\left(\mathrm{CD}^{-} 7^{-} / \mathrm{IgD}^{+} / \mathrm{IgM}^{ \pm} / \mathrm{CD} 38^{\text {low }}\right.$; Figure $\left.2 \mathrm{~B}\right)$.

These data confirm that subset 4 IGs have a favored reactivity for viable human B lymphocytes. Furthermore, the data indicate that subset $4 \mathrm{mAbs}$ bind both naïve and memory B cells. This latter finding differs from the nonstereotyped IGHV4-34 mAb tested (141) and the mAbs (30) and pAbs described by others in other contexts (31-33) that preferentially react with naïve B cells (34).

\section{The Cell Surface Autoantigenic Target of CLL Subset $4 \mathrm{mAbs}$ Is Distinct from the I/i Carbohydrate Antigens}

It is well documented that many IGHV4-34-encoded antibodies bind to poly- $N$-acetyllactosaminoglycans (NALs) of the i- and I- antigen type (35) found on the surfaces of human erythrocytes (36) and lymphocytes $(31,37)$. On human B lymphocytes, the autoantigenic epitope is predominantly a linear NAL of $\mathrm{i}$ antigen type present on CD45 (38-40). We therefore tested if CLL subset $4 \mathrm{mAbs}$ react with $\mathrm{CD} 45^{+}$and $\mathrm{CD} 45^{-}$cells of the $B$ cell lineage.

To do this, we used U266, a myeloma cell line, which, although predominantly 
A

A

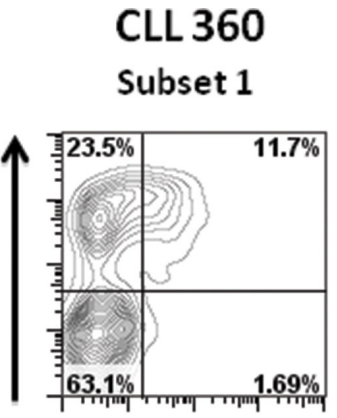

CLL 141 4-34; no subset

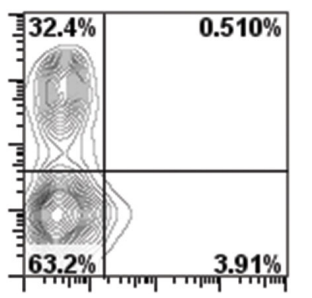

CLL 183 Subset 4

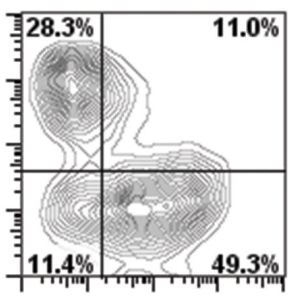

CLL 240 Subset 4

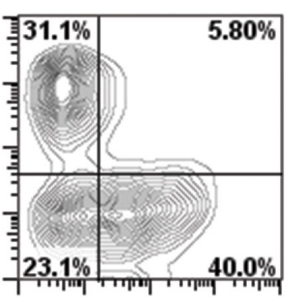

CLL 342 Subset 4

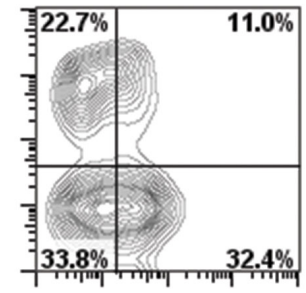

\section{CLL mAb}

B

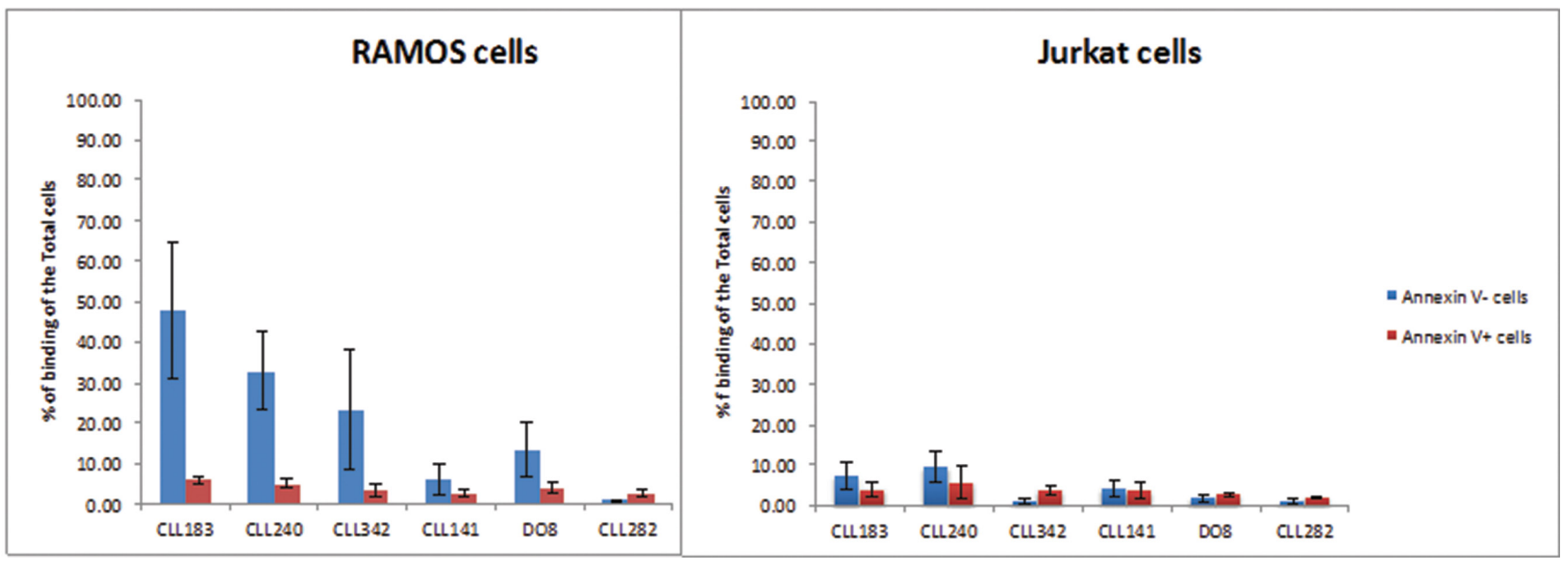

Figure 1. Subset $4 \mathrm{IGs}$ bind to viable human B lymphocytes. Subset 4 mAbs (nos. 183, 240, 342) were incubated with Ramos or Jurkat cell line cells under nonpermeabilizing conditions. mAb141, which expresses IGHV4-34 but does not belong to subset 4, and mAb360, which is a member of subset 1 and does not express IGHV4-34, were used as comparators. Apoptosis was measured by annexin $V$ staining. (A) Representative contour plots of CLL mAb binding to Ramos cells. Binding detected by FITC-labeled F(ab')2 fragments of goat polyclonal antibodies (pAbs) specific for human IgG. (B) Average binding of live (annexin $V^{-}$) and apoptotic/dead (annexin $V^{+}$) Ramos and Jurkat cells by the following biotinylated CLL mAbs: subset 4 mAbs 183, 240 and 342; non-subset 4 IGHV4-34-expressing CLL mAbs 141 and DO8; and subset 2 mAb282 (IGHV3-21/IGLV3-21). Binding was detected by streptavidin PE-Cy7. Data obtained from four independent experiments. Error bars represent SEM.

CD45-, has a definable subpopulation of $\mathrm{CD} 45^{+}$cells. After exposing U266 cells to mAb240, we observed that the mAb bound both the CD45- and $\mathrm{CD} 45^{+}$populations within the clone (Supplementary Figure 1). This was the case even when we tested binding to U266 after presorting the cell line into $\mathrm{CD} 45^{+}$and $\mathrm{CD} 45^{-}$ subpopulations (data not shown). Thus, the autoantigenic target for subset 4 is not limited to the CD45 antigen and need not be the linear NAL i epitope.

Next, we directly tested binding of the subset $4 \mathrm{mAbs}$ to a microarray of blood group-related carbohydrate sequences. Figure 3 illustrates microarray binding data for the 3 CLL subset 4 mAbs (183, 240 and 342), the IGHV4-34 ${ }^{+}$ non-subset 4 mAb141 and a control CLL subset 2 (IGHV3-21/IGLV3-21) mAb282, each at a $10 \mu \mathrm{g} / \mathrm{mL}$ concentration. These IGs were used in their normal bivalent state as well as multimers, created by preincubation with anti-human IgG pAbs. The microarray included the two linear i-active probes with type 2 (Gal $\beta$-4GlcNAc-) termini: the hexasaccharide NGL pLNnH,
Gal $\beta-4 G l c N A c \beta-3 G a l \beta-4 G l c N A c \beta-$ $3 \mathrm{Gal} \beta-4 \mathrm{Glc}-\mathrm{DH}$, and the octasaccharide NGL LNnO, Gal $\beta-4$ GlcNAc $\beta-3 G a l \beta-$ 4 GlcNAc $\beta$-3Gal $\beta$-4GlcNAc $\beta$-3Gal $\beta$ $4 \mathrm{Glc}-\mathrm{DH}$. Also included was a branched I-active probe with two type 2 termini, one of which is linked to the C6 position of the polylactosamine backbone, Gal $\beta-4 G l c N A c \beta-6(G a l \beta-4 G l c N A c \beta-3)$ Gal $\beta-4$ GlcNAc $\beta-3 G a l \beta-4 G l c-C e r$. The isomeric hexasaccharide glycan probe pLNH with type 1 (Galß-3GlcNAc-) terminus, Gal $\beta$-3GlcNAc $\beta$-3Gal $\beta$ 4 GlcNAc $\beta$-3Gal $\beta$-4Glc-DH, which lacks 
A

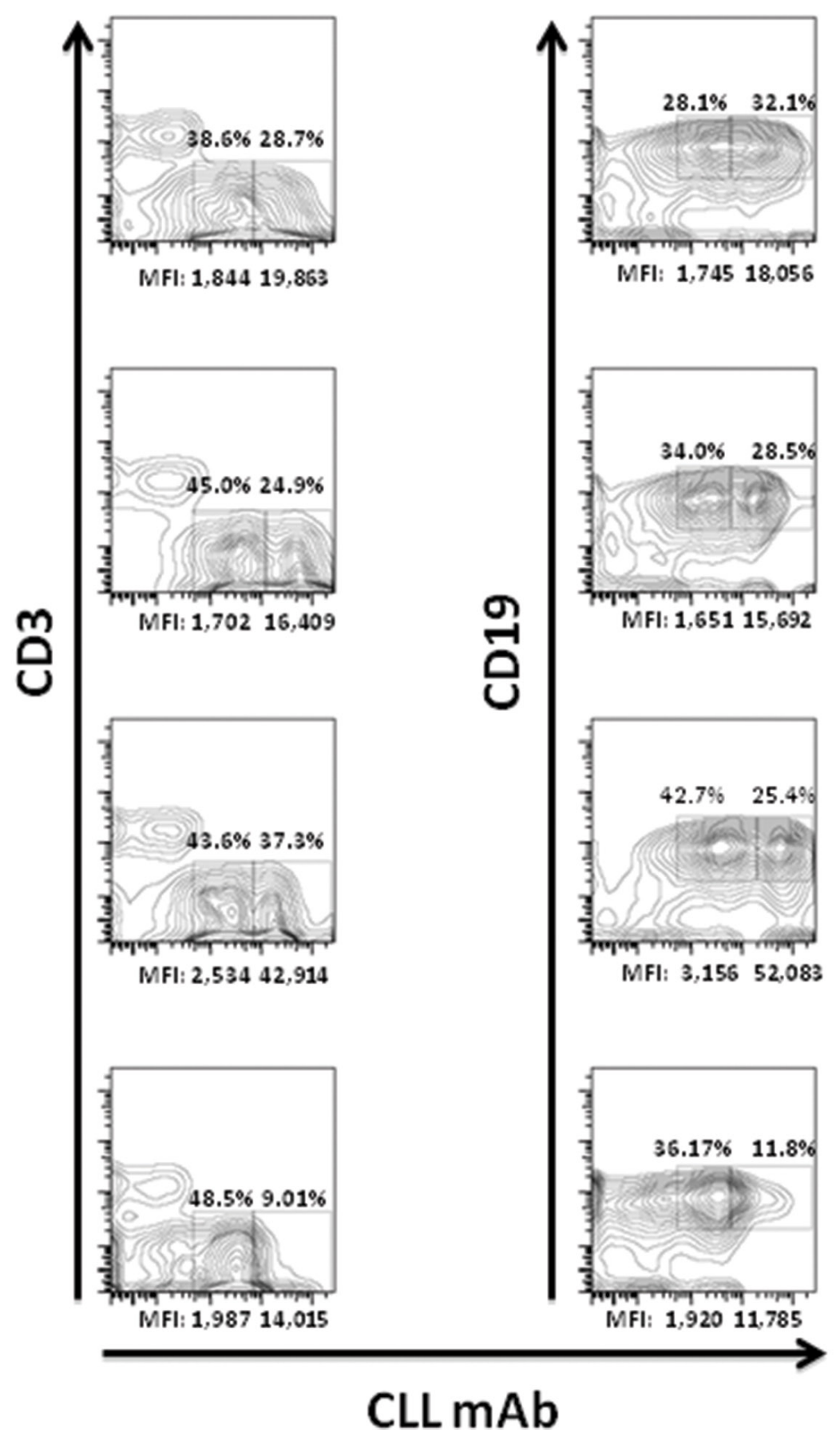

$\leftarrow$ mAb183

(Subset 4)

$\leftarrow \mathrm{mAb} 240$

(Subset 4)

$\leftarrow$ mAb342

(Subset 4)

$\leftarrow \mathrm{mAb} 141$

(4-34; no subset)

Figure 2. CLL IGs belonging to subset 4 bind with different intensities to memory and naïve B cells. Tonsil mononuclear cells were incubated with CLL subset 4 mAbs 183, 240 and 342, and with a panel of commercial antibodies reactive with membrane antigens that distinguish human B cell subsets. (A) Two subpopulations (dim and bright) of CD19+ cells can be identified based on CLL subset 4 mAb binding. mAb141, which uses IGHV4-34 but does not belong to subset 4, recognizes only one population. (B) After gating specifically on $\mathrm{CD} 19^{+}$cells, it is clear that the CD19+/CLL mAbright fraction is predominantly $\mathrm{CD}^{\text {b }} 7^{-} / \mathrm{lgD} / \mathrm{CD}^{+} 8^{\text {low }}$ (nailve $\mathrm{B}$ cells) and the $\mathrm{CD} 19^{+} / \mathrm{CLL} \mathrm{mAb} \mathrm{b}^{\text {dim }}$ cells are mainly $\mathrm{CD} 27^{+} / \mathrm{lgD}^{-} / \mathrm{CD} 38^{\text {low/high }}$ (memory B cells). In contrast, CD $19^{+}$cells bound by mAb141 are predominantly $\mathrm{CD}_{27^{-} / \mathrm{lgD}} / \mathrm{CD} 38^{\text {low }}$ cells (naïve B cells). 
B

mAb183

(Subset 4)
mAb240

(Subset 4)
mAb342

(Subset 4)
mAb141

(4-34; non subset)

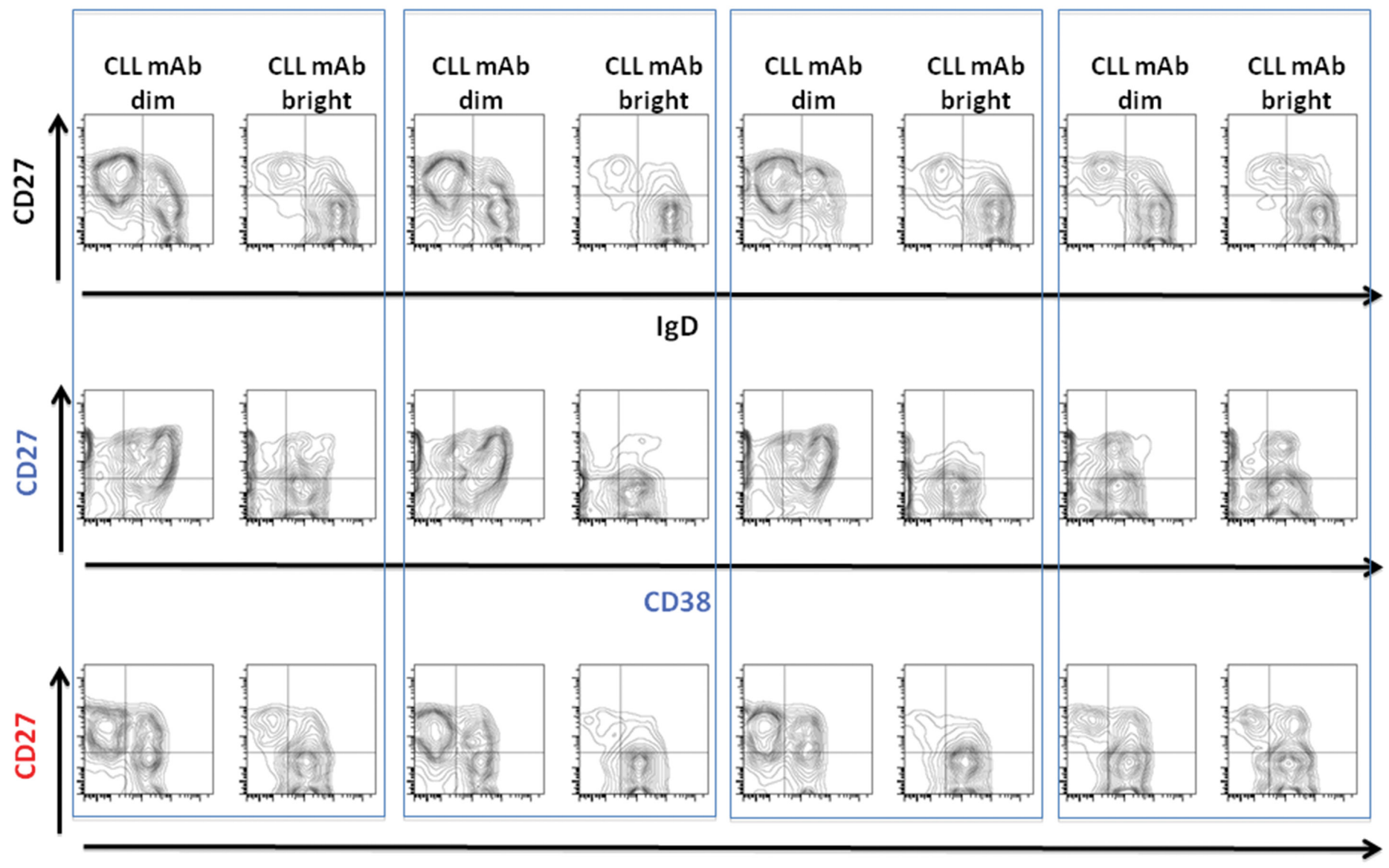

IgM

Figure 2. Continued.

i-type antigen reactivity but expresses an antigen recognized by the murine IgM mAb Fc10.2 directed at fetal endoderm, was also included (Figure 3A). The subset $4 \mathrm{mAbs}$ did not bind to the i- and I-type glycans, nor the FC10.2 antigen analog (Figure 3B), nor to other blood group-related sequences in the array (results not shown). Moreover, the CLL IGs did not react with these targets if precomplexed to create multimeric structures resembling IgM (Figure 3C). However, IGHV4-34 $4^{+}$CLL mAb141, bivalent or multimeric, bound well to the representative i-antigenexpressing probes in the array, pLNnH and LNnO. As controls, human sera containing anti-i (P1A ELL) and anti-I
(Ma) IgM antibodies and murine IgM mAb Fc10.2 $(27,28)$ reacted well with their respective target carbohydrate antigens: the anti-i with pLNnH and LNnO, the anti-I with I-octa and Fc10.2 with $\mathrm{pLNH}$. These findings validate the structures of the poly LacNac sequences displayed on the array.

Together, the above results indicate that CLL subset 4 mAbs have an autoreactivity distinct from that described in other malignant and autoimmune conditions $(13-16,23)$, since they do not interact with the carbohydrate autoantigen classically recognized by nonstereotyped IGHV4-34 IGs. In this regard, the non-subset 4 IGHV4-34 ${ }^{+}$mAb141 binds the classical epitopes (Figure 3).

\section{CLL Subset 4 IGs Do Not Bind DNA or Other Intracellular Autoantigens}

Next, we tested the reactivity of subset 4 mAbs with nuclear and cytoplasmic autoantigens by exposing the three CLL mAbs to HEp-2 cells and detecting binding using goat anti-human IgG pAbs conjugated with a fluorochrome. The three subset $4 \mathrm{mAbs}$ showed negligible binding to permeabilized HEp-2 cells compared with a subset $8 \mathrm{mAb}$ (no. 114; IGHV4-39/IGKV1(D)-39) that binds well to cytoplasmic structures and compared with a non-subset $\mathrm{mAb}$ (no. 255) that binds nuclear constituents (Figure 4A). The lack of binding to nuclear structures within HEp-2 cells is inconsistent with the previously described 
A

pLNH:

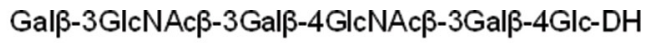

pLNnH:

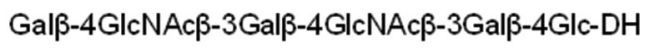

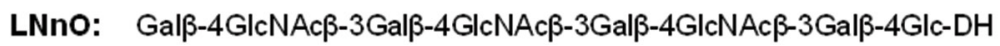

I-octa:

Gal $\beta-4 G \mid c N A c \beta-6$
Gal $\beta-4 G\left|c N A c \beta-{ }_{3}^{G a l} \beta-4 G\right| c N A c \beta-3 G a l \beta-4 G l c-C e r$

B

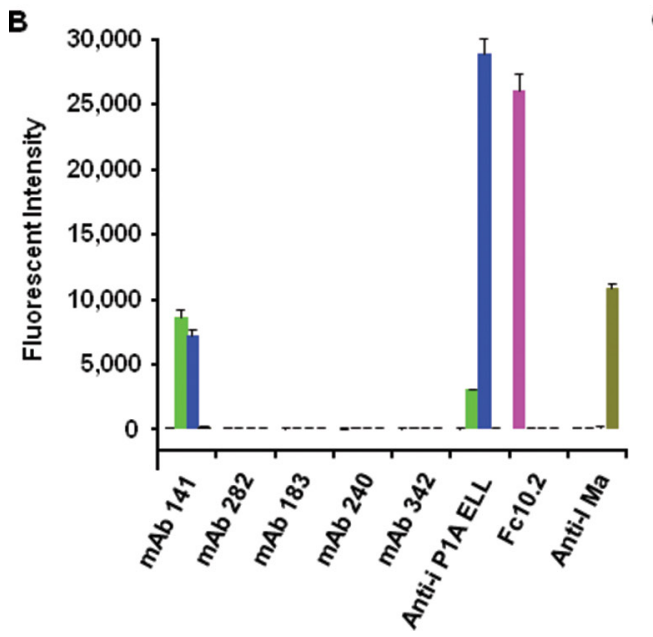

C

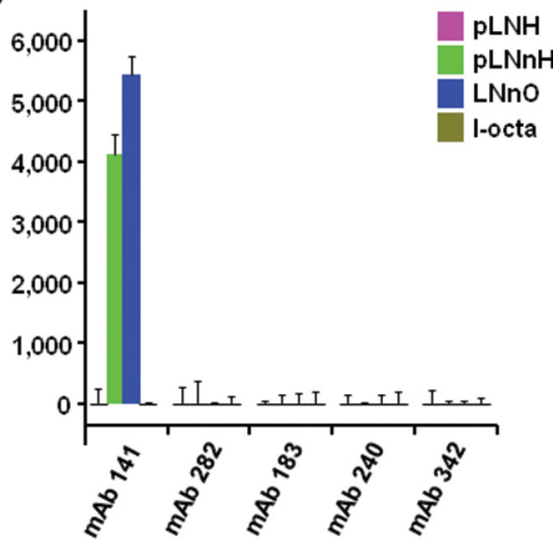

Figure 3. CLL subset 4 IGs do not bind i or I carbohydrate antigens. (A) Glycan sequences of the lipid-linked oligosaccharides pLNH, pLNnH, LNnO and l-octa that were among 76 additional (i- and Fc 10.2- antigen-negative probes) in the microarray. Abbreviations: Gal, galactose; GIcNAc, N-acetylglucosamine; Glc, glucose; DH, L-1,2-dihexadecyl-sn-glycero3-phosphoethanolamine; Cer, ceramide. (B), (C) Microarray analysis of CLL MAbs 141, $282,183,240$, and 342 , in either (B) native bivalent or (C) precomplexed condition. The human anti-i IgM PIA ELL, human anti-I Ma, and the murine IgM Fc 10.2 were included as positive controls. The results are the means of fluorescence intensities of duplicate spots, printed at $5 \mathrm{fmol}$, with error bars representing half the difference between the two values.

reactivity with DNA and other nucleic acid-protein conjugates defined for nonstereotyped IGHV4-34-expressing antibodies in autoimmune settings (31-33). This lack of DNA binding was confirmed directly by ELISA (Figure 4B). Thus, an antigenic reactivity that would be expected of IGHV4-34-expressing human Abs is absent in CLL subset 4 IGHV4-34 $4^{+} \mathrm{mAbs}$.

\section{Initial Studies to Determine the Target Antigen of CLL Subset 4 mAbs on Viable Human B Cell Surfaces}

To examine structural aspects of subset 4 antibody binding, we attempted to inhibit binding to viable human B cells by preincubating the CLL IGs with the IGHV4-34-reactive $\mathrm{mAb}, 9 \mathrm{G} 4$. This did not result in an appreciable decrease in binding. However, preliminary immunofluorescence experiments comparing binding of CLL mAb240 to the Ramos cell line cultured in the presence or absence of inhibitors of the synthesis of $\mathrm{N}$-glycans (tunicamycin) or O-glycans (benzyl 2-acetamido-2-deoxy- $\alpha$-Dgalactopyranoside, BADGP) or glycolipids (D-1-phenyl-2-hexadecanoylamino3-morpholino-1-propanol, PPMP) suggest that the target of the subset 4 CLL IG $\mathrm{mAb} 240$ is carried on N-glycans (data not shown). Detailed studies are under way to corroborate this possibility and establish the structure of the determinant.

\section{Structural Features of Subset 4 IGKV- IGKJ Gene Rearrangements that Permit Binding to Viable Human Memory and Naïve B Cells}

Since subset $4 \mathrm{mAbs}$ recurrently develop replacement mutations that create specific amino acids at defined sites / positions within the portion of the IG heavy $(\mathrm{H})$ and light $(\mathrm{L})$ variable domains coded by the IGHV4-34 and IGKV2-30 genes (14) (Figure 5), we investigated the roles of the individual somatically generated amino acids on binding to viable human B cells. For these studies, we used CLL subset $4 \mathrm{mAb} 240$ as the starting template and selectively and specifically transformed its amino acid sequence back to that corresponding to the germline sequence of the IGHV4-34 and IGKV2-30 genes. Specifically, for IGHV4-34, we reverted four single amino acid mutations, N22T (rH-1), E28G (rH-2), A99T ( $\mathrm{rH}-3)$ and I101V ( $\mathrm{rH}-4)$, and a final revertant, $\mathrm{rH}-5$, that had all four individual changes together. For IGKV2-30, we made one revertant, D66N (rL-1), since this gene differed from the corresponding germline sequence at only this residue.

Note that some of the positions reverted to the germline equivalent are frequently mutated in subset $4 \mathrm{mAbs}$; for example, amino acids at codon 28 in the VH CDR1 of IGHV4-34 and at codon 66 in the VK FR3 of IGKV2-30 (Figure 5 and ref. 14). The other mutations found in $\mathrm{mAb} 240$ are unique to this $\mathrm{mAb}$ and are not frequently seen in other members of subset 4 .

After incubating the altered versions of mAb240 containing each of the IGHV4-34 H chain revertants with viable $\mathrm{B}$ cells from tonsil samples and analyzing them by flow cytometry, no substantial reproducible change in binding was observed from that using the original wildtype (wt) $\mathrm{H}$ chain (not shown). This was the case if we altered only single amino acids, including the "subset 4-biased" mutation at position 28 in VH CDR1, or all four mutations. Similar results were 
A

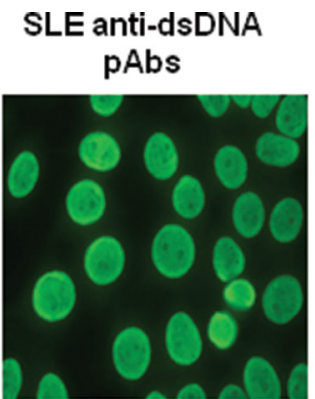

CLL183

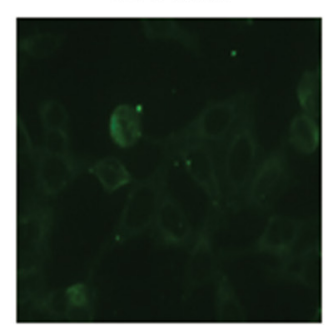

CLL IGHV4-39 subset 8 mAb114

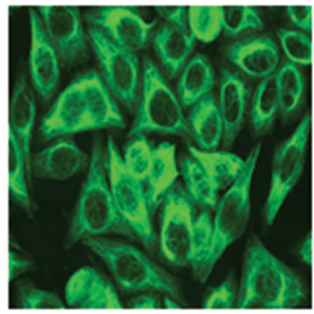

CLL240

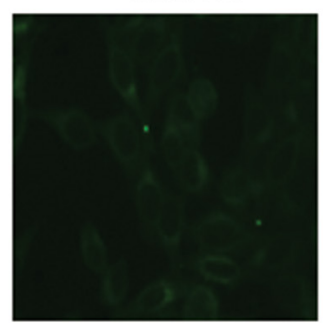

CLL IGHV4-59 non-subset mAb255

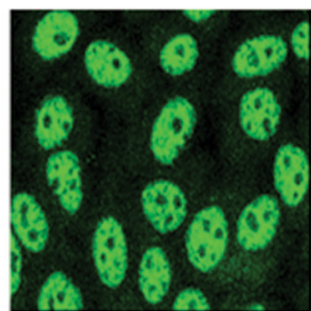

CLL342

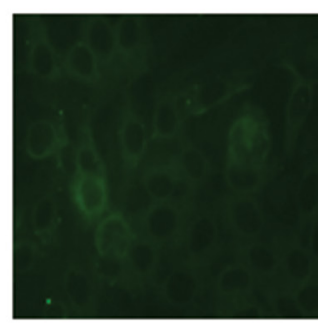

B

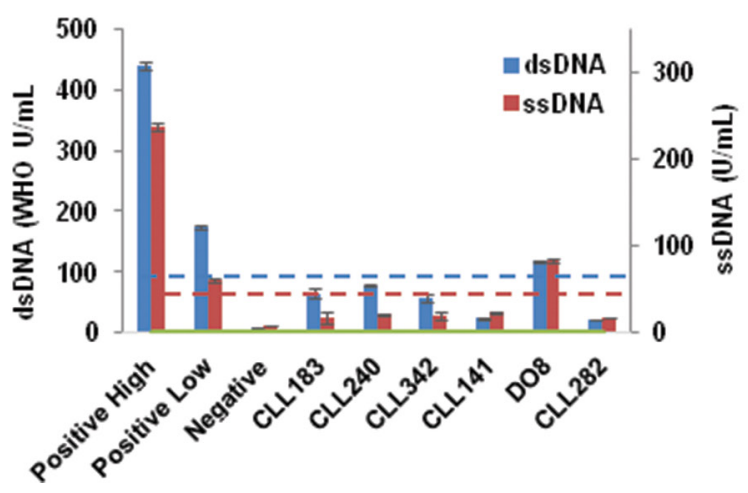

Figure 4. Binding of subset 4 IGs to permeabilized and fixed HEp-2 cells and to DNA. (A) Reactivity of subset 4 IGs with permeabilized and fixed HEp-2 cells. Binding of Abs was detected using fluorochrome-labeled goat anti-human IgG pAbs. (B) Binding of native CLL IGs to DNA determined by ELISA. IGHV4-34+ subset 4 mAbs (nos. 183, 342 and 240), IGHV4-34+ non-subset 4 mAbs (nos. 141 and DO8) and subset 2 (IGHV3-21/IGLV3-21) mAb (no. 282) were tested for reactivity with dsDNA and ssDNA by enzyme immunoassay. Antibodies were used at a concentration of $25 \mathrm{ug} / \mathrm{mL}$. Human sera containing antibodies reactive with dsDNA or with ssDNA were used as positive controls. Human serum without antibodies against dsDNA or ssDNA was used as negative control. Data are average \pm SD of at least three independent experiments. Following the calculation and result interpretation information provided by the manufacturer, values $\geq 92.6 \mathrm{WHO}$ units $/ \mathrm{mL}$ for dsDNA binding (dashed blue line) and $\geq 68.6 \mathrm{U} / \mathrm{mL}$ for ssDNA binding (dashed red line) were registered as positive.

obtained when testing Ramos B cell line cells (not shown).

Therefore, we next analyzed the role of the L chain revertant at codon 66 in the VK FR3 on the binding of subset 4 mAb240 to healthy B cells from tonsil (Figure 6A). To do this, we paired the revertant $\mathrm{L}$ chain with the wt $\mathrm{H}$ chain (240Hwt + rL-1), and then analyzed binding of this and the unmanipulated mAb240 by flow cytometry. When the $\mathrm{mAb}$ containing the $\mathrm{rL}-1$ revertant was used, binding of viable $B$ cells decreased significantly (Figure 6A, orange line), compared with binding of mAb240 with the wt L chain (Figure 6A, blue line). Furthermore, the revertant now bound mainly the $\mathrm{CD} 19^{+} / \mathrm{CLL}$ mAb ${ }^{\text {bright }}$ cells (Figure 6A, contour plot) that have the phenotype of $\mathrm{CD}^{-} 7^{-} / \mathrm{IgD}^{+}$naïve $\mathrm{B}$ cells (Figure 2B). Similarly, we observed a marked decrease of $\mathrm{B}$ cell binding when staining Ramos cells with the L chain revertant-containing mAb240 (Figure 6B, left panel, orange line). Indeed, the extent of change in antigen binding observed with a single amino acid change in the $\mathrm{L}$ chain of $\mathrm{mAb} 240$ is equal to that seen when a completely different L chain (from non-subset $4 \mathrm{mAb} 141$ that uses IGKV1-27) is paired with the $\mathrm{H}$ chain of the other subset $4 \mathrm{mAb} 183$ (Figure 6B, right panel, orange line).

Hence, reactivity of subset $4 \mathrm{mAbs}$ with viable human $B$ cells and with memory B cells is a function of the somatically generated $D$ residue at position 66 of the L chain that is characteristic of this stereotyped CLL subset. This structural alteration involves a change of an amino acid in the FR and not the CDR portion of the $\mathrm{mAb}$. Of note, the amino acid change introduces a charged residue at this site.

\section{DISCUSSION}

In this study, we investigated antigen binding by recombinant $\mathrm{mAbs}$ from patients belonging to CLL stereotyped subset 4 , the largest M-CLL subset and a prototype for indolent disease. The binding of these subset 4 mAbs was compared with other Abs using the IGHV4-34 gene in nonstereotyped configurations. Our findings are informative not only for the specific reactivities we identified, but also for those that were expected based on the literature (31-33) that we did not find. Specifically, we documented that, unlike most CLL mAbs (stereotyped or not), subset 4 IGHV4-34-expressing mAbs do not bind to the surface of apoptotic human B lymphocytes; rather, they bind selectively to viable cells. Furthermore, after conducting binding studies with normal B cell subsets, we discovered 
A

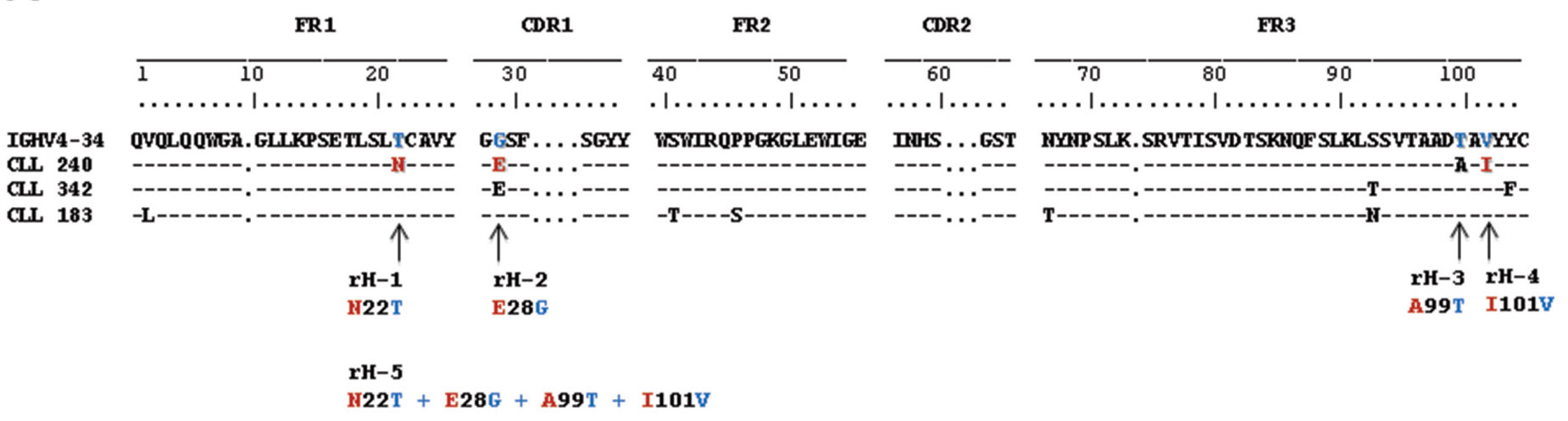

\section{B}

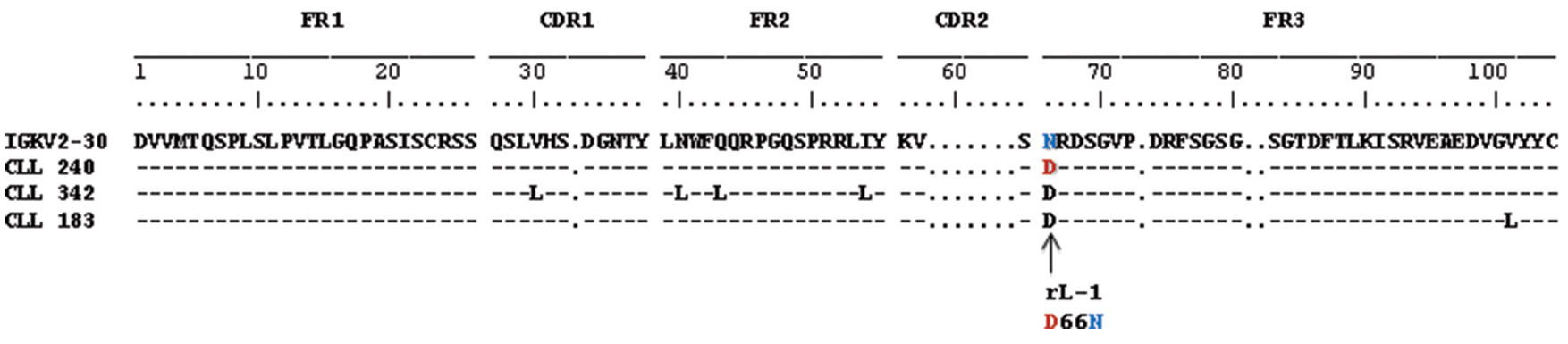

Figure 5. Sequences of the IGHV-D-J and IGKV-J rearrangements of subset 4 IGs and of the various IGHV4-34 and IGKV2-30 point revertants. The (A) IGHV4-34- and (B) IGKV2-30-encoded parts of the IG H and L variable domains of CLL subset 4 mAbs (nos. 240,342 and 183) are aligned with the corresponding germline counterpart. $\mathrm{H}$ and $\mathrm{L}$ chain revertants are indicated by arrows.

that subset 4 IGs bind both naïve and memory B lymphocytes, unlike IGHV4$34^{+}$Abs in other disease settings that bind exclusively to naïve B cells (34). In addition, and unlike most reported IGHV4-34-expressing Abs, CLL subset 4 $\mathrm{mAbs}$ do not react with DNA, as IGHV4-34 ${ }^{+}$Abs often do in systemic autoimmunity (31-33), nor with i-active NALs, as many IGHV4-34-expressing mAbs do in cold agglutinin disease (30). Thus, the uniqueness of the subset 4 IGHV434/IGHD5-18/IGHJ6 + IGKV2-30/IGKJ2 rearrangements overrides the ability of IGHV4-34 germline-encoded structures to bind DNA and i/I NALs and imparts reactivity with an antigen present on the surface of a defined functional subset of healthy human B cells. Moreover, to bind the B cell epitope, a residue (D66N) not encoded by the germline sequence is required in FR3; this lies at the junction of VK CDR2.

Although in principle it is possible that any germline $I G H V$ could react with any antigen when associated with an appropriate IGHD and IGHJ as well as an IGK/LVIGK/LJ rearrangement, IGHV4-34 appears to be unique among most germline genes in that it is inherently biased to react with autoantigens (41). This notion is strongly supported by the exclusion of normal human B lymphocytes bearing the stamp of IGHV4-34 rearrangements from entering germinal centers (42), where somatic hypermutation and class switch recombination can render innately autoreactive gene products even more dangerous by selecting variants having higher-avidity autoantigen-binding sites and/or more efficient IG effector isotypes. In this light, the lack of reactivity of CLL subset $4 \mathrm{mAbs}$ to DNA and $\mathrm{i}$ antigen is remarkable. The inability to bind these two purified autoantigens was shown directly in solid-phase antigen binding assays using multiple subset 4 mAbs (Figures 3 and 4) that were selected for testing based solely upon available DNA sequences. Moreover, the amino acid residues known to be responsible for binding the i-type NAL antigen (W at position 7 and AVY at positions 24, 25 and 26; Figure 5) are not altered in the subset 4 IGs studied here and in most subset $4 \mathrm{mAbs}$ in available databases $(11,14)$.

It is possible that a protein prepared recombinantly does not fold like the native molecule made in a cell. Short of conducting a study of the crystal 
A

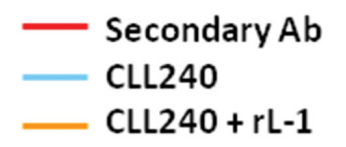

$\mathrm{rL}-1=\mathrm{D} 66 \mathrm{~N}$ revertant

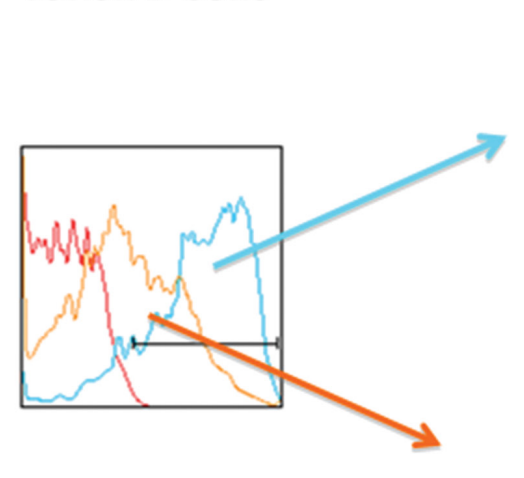

Tonsil B cells

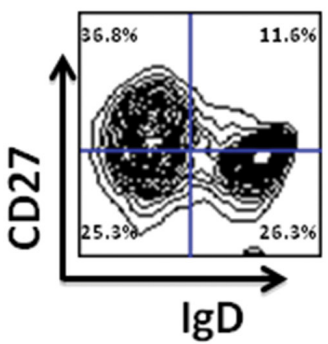

Memory and naïve $B$ cell reactivity of CLL240 with original IGKV2-30

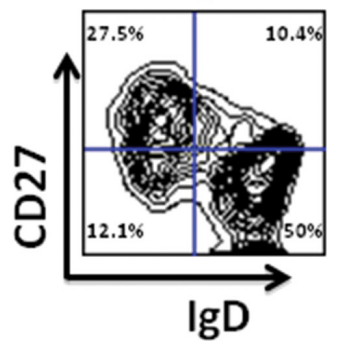

Memory and naïve $B$ cell reactivity of CLL240 with reverted IGKV2-30

B

\section{Ramos B cells}
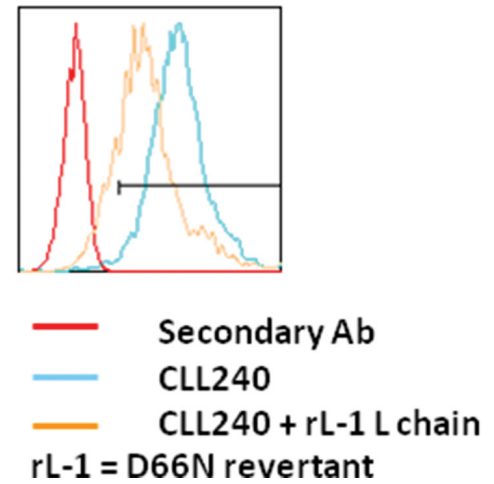
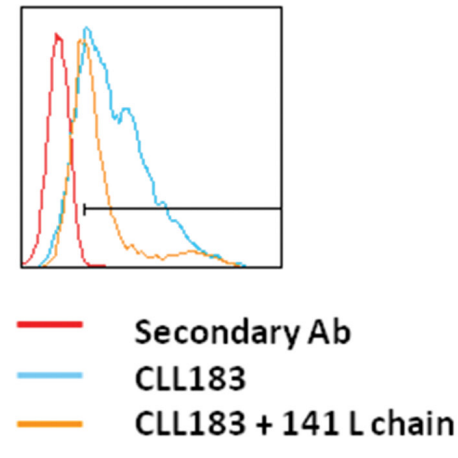

Figure 6. Effect of individual IGHV4-34 reversions on the binding of subset $41 G 240$ to viable human $B$ cells. (A) Effect of light chain reversion on binding to tonsil B lymphocytes. Tonsil mononuclear cells were incubated with biotinylated versions of CLL subset $4 \mathrm{mAb} 240$ and the original $240 \mathrm{H}$ chain paired with the L chain revertant $\mathrm{rL}-1$, followed by incubation with streptavidin PE-Cy7. Note the significant drop in memory B cell binding and the increase in naïve B cell binding. (B) Effect of light chain reversion or light chain swapping on binding to Ramos B cells. Ramos B cell line cells were incubated with unmanipulated CLL subset $4 \mathrm{mAb} 240$ or the original $240 \mathrm{H}$ chain paired with the $\mathrm{L}$ chain revertant $\mathrm{rL}-1$ (left panel) or with unmanipulated $\mathrm{CLL}$ subset $4 \mathrm{mAb} 183$ or the original $183 \mathrm{H}$ chain paired with the $\mathrm{L}$ chain rearrangement from CLL mAb 141, an IGHV4-34+ IG that is not a member of subset 4 (right panel). In both cases (left and right panels), each $\mathrm{mAb}$ was followed by incubation with streptavidin PE-Cy7. See Figure 5 for explanation of abbreviation for rL-1 revertant.

structure of the CLL mAbs used here, this possibility cannot be excluded. However, we do know that the $\mathrm{H}$ chain is associated with the provided L chain, as both are found on sodium dodecyl sulfate polyacrylamide gel electrophoresis run under reducing conditions. Also the $\mathrm{H}$ and $\mathrm{L}$ chain complex has an appropriate molecular weight based on mobility in size exclusion chromatography (data not shown). The lack of binding of these antibodies to I/i-type NALs requires further investigation with a wider range of carbohydrates.

A case for positive selection of a novel antigen-binding site is strengthened by our finding that subset $4 \mathrm{mAbs}$ do not react with apoptotic human $B$ cells, while they do bind to viable B lymphocytes (Figure 1). Furthermore, this disparity suggests that the target antigen on the $B$ cell surface is present constitutively and therefore differs from target antigens brought to the cell surface from the inside on apoptotic cells. This selective binding to viable and not apoptotic cells also suggests that the antigenic epitope is modified, destroyed or removed by internalization or cleavage during the apoptotic process, consistent with structural antigenic changes due to oxidation $(20,43)$, flipping of membrane components (44) or enzymatic cleavage $(45,46)$. 
The second distinguishing feature of subset $4 \mathrm{mAb}$ binding to viable human B cells is reactivity with two phenotypically distinct B cell subsets with different functional properties. Specifically, subset 4 IGs bind memory and naïve human B cells. Although the antigenic epitope recognized could be shared by the two B cell subsets, two findings suggest that this is not the case. First, mAb141, which uses the IGHV4-34 gene but not the IGHD, $I G H J$ or IGKV genes of subset 4, binds to naïve $B$ cells and not to memory B cells. We predict that this is due to reactivity with i-antigen, as indicated in Figure 3. Second, reversion of the subset 4 characteristic somatically generated charged residue (aspartic acid, D) to the germline-encoded residue (asparagine, N) at position 66 in the VK FR3 of the kappa L chain significantly reduces binding to memory $B$ cells and enhances reactivity with naïve $B$ cells (Figure 6A). Even though this obligate aspartic acid resides in the VK FR3, it is the first residue after VK CDR2, suggesting that it might influence the structure or accessibility of the VK CDR2 to antigen binding. The somatic nature of this residue highlights that the CLL precursor B cell was not excluded from undergoing a germinal center reaction, as is the case for most normal human B lymphocytes (42).

In CLL, the functional consequences of subset 4 binding to memory and naïve $B$ cells have yet to be determined. In other instances, anti-lymphocyte Abs, some of which can be lymphocyte subsetspecific $(47,48)$, cause cytolysis $(49)$. This has also been described for certain IGHV4-34 $4^{+} \mathrm{mAbs}$ (50). Therefore, the indolent clinical course of CLL subset 4 patients (51) could relate to an ongoing immunoregulatory interaction of these B cells and their Ig products. By extension, we could speculate that such an interaction might also induce and/or maintain the anergic status of subset 4 CLL cells (18), thus lying at the core of diminished responsiveness to external triggering that characterizes this prototypic favorable prognostic CLL subgroup.

\section{ACKNOWLEDGMENTS}

This work was supported in part by an RO-1 grant from the National Cancer Institute/National Institutes of Health (CA081554) (to NC), and Bloodwise grant 14028 and Wellcome Trust grants WT093378MA and WT099197MA (to TF). Additional support in the form of philanthropic contributions was provided by the Karches Foundation, Marks Foundation, Nash Family Foundation, Jerome Levy Foundation, Leon Levy Foundation, Frank and Mildred Feinberg Foundation, Mona and Edward Albert Foundation and Jean Walton Fund for Leukemia, Lymphoma, and Myeloma Research.

\section{CONCLUSION}

Using recombinant IGs corresponding to CLL clones expressing IGHV4-34 in either stereotyped subset 4 or nonstereotyped configurations, we have shown that CLL subset 4 BCR IGs do not bind DNAs nor i or I carbohydrate antigens; these are common targets of IGHV4-34-utilizing antibodies in systemic lupus erythematosus and cold agglutinin disease, respectively. However, these IGs do react with viable memory B cells as well as naïve B lymphocytes, the latter being bound by other nonsubset 4, IGHV4-34-utilizing antibodies. Notably, reactivity with viable B cells depends on the acquisition of a somatic mutation at position 66 of FR3 in the rearranged IGKV2-30 gene. This mutation changes a hydrophilic, uncharged amino acid (asparagine) to an acidic amino acid (aspartic acid) at that site. Our findings illustrate the importance of positive and negative selection for acquired structural changes in CLL subset 4 IGs in determining the unique antigen-reactivities of these CLL clones. These findings also suggest that the autoantigens driving normal B cells to become subset 4 CLL cells differ from those driving IGHV4-34-expressing B cells in other malignancies and in autoimmune diseases.

\section{DISCLOSURE}

The authors declare that they have no competing interests as defined by Molecular Medicine or other interests that might be perceived to influence the results and discussion reported in this paper.

\section{REFERENCES}

1. Chiorazzi N, Ferrarini M. (2003) B cell chronic lymphocytic leukemia: lessons learned from studies of the B cell antigen receptor. Annu. Rev. Immunol. 21:841-94.

2. Chiorazzi N, Rai KR, Ferrarini M. (2005) Chronic Lymphocytic Leukemia. N. Engl. J. Med. 352(8):804-15.

3. Packham G, Stevenson F. (2010) The role of the B-cell receptor in the pathogenesis of chronic lymphocytic leukaemia. Sem. Cancer Biol. 20(6):391-99.

4. Hashimoto S, et al. (1995) Somatic diversification and selection of immunoglobulin heavy and light chain variable region genes in IgG+ CD5+ chronic lymphocytic leukemia B cells. J. Exp. Med. 181(4):1507-17.

5. Tobin G, et al. (2003) Chronic lymphocytic leukemias utilizing the VH3-21 gene display highly restricted V\{lambda\}2-14 gene use and homologous CDR3s: implicating recognition of a common antigen epitope. Blood. 101(12):4952-57.

6. Ghiotto F, et al. (2004) Remarkably similar antigen receptors among a subset of patients with chronic lymphocytic leukemia. J. Clin. Invest. 113(7):1008-16.

7. Messmer BT, et al. (2004) Multiple distinct sets of stereotyped antigen receptors indicate a role for antigen in promoting chronic lymphocytic leukemia. J. Exp. Med. 200(4):519-25.

8. Tobin G, et al. (2004) Subsets with restricted immunoglobulin gene rearrangement features indicate a role for antigen selection in the development of chronic lymphocytic leukemia. Blood. 104(9):2879-85.

9. Stamatopoulos K, et al. (2007) Over $20 \%$ of patients with chronic lymphocytic leukemia carry stereotyped receptors: Pathogenetic implications and clinical correlations. Blood. 109(1):259-70.

10. Darzentas N, et al. (2010) A different ontogenesis for chronic lymphocytic leukemia cases carrying stereotyped antigen receptors: molecular and computational evidence. Leukemia. 24(1):125-32.

11. Agathangelidis A, et al. (2012) Stereotyped B-cell receptors in one-third of chronic lymphocytic leukemia: a molecular classification with implications for targeted therapies. Blood. 119(19):4467-75.

12. Fais F, et al. (1998) Chronic lymphocytic leukemia B cells express restricted sets of mutated and unmutated antigen receptors. J. Clin. Invest. 102(8):1515-25.

13. Vardi A, et al. (2014) Immunogenetic Studies of Chronic Lymphocytic Leukemia: Revelations and 
Speculations about Ontogeny and Clinical Evolution. Cancer Res. 74(16):4211-6.

14. Murray F, et al. (2008) Stereotyped patterns of somatic hypermutation in subsets of patients with chronic lymphocytic leukemia: implications for the role of antigen selection in leukemogenesis. Blood. 111(3):1524-33.

15. Sutton LA, et al. (2009) Extensive intraclonal diversification in a subgroup of chronic lymphocytic leukemia patients with stereotyped IGHV4-34 receptors: implications for ongoing interactions with antigen. Blood. 114(20):4460-68.

16. Baliakas P, et al. (2014) Recurrent mutations refine prognosis in chronic lymphocytic leukemia. Leukemia. 29(2):329-36.

17. Muzio M, et al. (2008) Constitutive activation of distinct BCR-signaling pathways in a subset of CLL patients: a molecular signature of anergy. Blood. 112(1):188-95.

18. Ntoufa S, et al. (2016) B Cell Anergy Modulated by TLR1/2 and the miR-17-92 Cluster Underlies the Indolent Clinical Course of Chronic Lymphocytic Leukemia Stereotyped Subset \#4. J. Immunol. 196(10):4410-7.

19. Cesano A, et al. (2013) Association between B-cell receptor responsiveness and disease progression in B-cell chronic lymphocytic leukemia: results from single cell network profiling studies. Haematologica. 98(4):626-34.

20. Catera R, et al. (2008) Chronic lymphocytic leukemia cells recognize conserved epitopes associated with apoptosis and oxidation. Mol. Med. 14(11-12):665-74.

21. Chu CC, et al. (2010) Many chronic lymphocytic leukemia antibodies recognize apoptotic cells with exposed nonmuscle myosin heavy chain IIA: implications for patient outcome and cell of origin. Blood. 115(19):3907-15.

22. Lanemo Myhrinder A, et al. (2008) A new perspective: molecular motifs on oxidized LDL, apoptotic cells, and bacteria are targets for chronic lymphocytic leukemia antibodies. Blood. 111(7):3838-48.

23. Wardemann H, et al. (2003) Predominant autoantibody production by early human B cell precursors. Science. 301(5638):1374-77.

24. Chai W, Stoll MS, Galustian C, Lawson AM, Feizi T. (2003) Neoglycolipid technology: deciphering information content of glycome. Methods Enzymol. 362:160-95.

25. Liu Y, et al. (2012) Neoglycolipid-based oligosaccharide microarray system: preparation of NGLs and their noncovalent immobilization on nitrocellulose-coated glass slides for microarray analyses. Methods Mol. Biol. 808:117-36.

26. Gao C, et al. (2014) Carbohydrate sequence of the prostate cancer-associated antigen $\mathrm{F} 77$ assigned by a mucin O-glycome designer array. J. Biol. Chem. 289(23):16462-77.

27. Gao C, Zhang Y, Liu Y, Feizi T, Chai W. (2015) Negative-Ion Electrospray Tandem Mass Spectrometry and Microarray Analyses of Developmentally Regulated Antigens Based on Type 1 and Type 2 Backbone Sequences. Anal. Chem. 87(23):11871-78.

28. Gooi HC, et al. (1983) A marker of human foetal endoderm defined by a monoclonal antibody involves type 1 blood group chains. Mol. Immunol. 20(6):607-13.

29. Palma AS, et al. (2006) Ligands for the beta-glucan receptor, Dectin-1, assigned using "designer" microarrays of oligosaccharide probes (neoglycolipids) generated from glucan polysaccharides. J. Biol. Chem. 281(9):5771-79.

30. Pascual V, et al. (1992) VH restriction among human cold agglutinins. The VH4-21 gene segment is required to encode anti-I and anti-i specificities. J. Immunol. 149(7):2337-44.

31. Grillot-Courvalin C, et al. (1992) An anti-B cell autoantibody from Wiskott-Aldrich syndrome which recognizes i blood group specificity on normal human B cells. Eur. J. Immunol. 22(7):1781-88.

32. Stevenson FK, et al. (1993) Utilization of the VH4-21 gene segment by anti-DNA antibodies from patients with systemic lupus erythematosus. J. Autoimmun. 6(6):809-25.

33. Borretzen M, Chapman C, Stevenson FK, Natvig JB, Thompson KM. (1995) Structural analysis of VH4-21 encoded human IgM allo- and autoantibodies against red blood cells. Scand. J. Immunol. 42(1):90-97.

34. Cappione AJ, Pugh-Bernard AE, Anolik JH, Sanz I. (2004) Lupus IgG VH4.34 Antibodies Bind to a 220$\mathrm{kDa}$ Glycoform of CD45/B220 on the Surface of Human B Lymphocytes. J. Immunol. 172(7):4298-307.

35. Smith G, Spellerberg M, Boulton F, Roelcke D, Stevenson F. (1995) The immunoglobulin VH gene, VH4-21, specifically encodes autoanti-red cell antibodies against the I or i antigens. Vox Sanguinis. 68(4):231-35.

36. Feizi T, Childs RA, Watanabe K, Hakomori SI. (1979) Three types of blood group I specificity among monoclonal anti-I autoantibodies revealed by analogues of a branched erythrocyte glycolipid. J. Exp. Med. 149(4):975-80.

37. Feizi T, Kapadia A, Yount WJ. (1980) I and i antigens of human peripheral blood lymphocytes cocap with receptors for concanavalin A. Proc. Natl. Acad. Sci. USA. 77(1):376-80.

38. Childs RA, Feizi T. (1981) Differences in carbohydrate moieties of high molecular weight glycoproteins of human lymphocytes of T and B origins revealed by monoclonal autoantibodies with anti-I and anti-I specificities. Biochem. Biophys. Res. Commun.102(4):1158-64.

39. Childs RA, et al. (1983) Evidence for the occurrence of O-glycosidically linked oligosaccharides of poly-N-acetyllactosamine type on the human leucocyte common antigen. Biochem. Biophys. Res. Commun. 110(2):424-31.

40. Silberstein LE, George A, Durdik JM, Kipps TJ. (1996) The V4-34 encoded anti-i autoantibodies recognize a large subset of human and mouse B-cells. Blood Cells Mol. Dis. 22(2):126-38.

41. Pugh-Bernard AE, et al. (2001) Regulation of inherently autoreactive VH4-34 B cells in the maintenance of human B cell tolerance. J. Clin. Invest. 108(7):1061-70.

42. Cappione A 3d, et al. (2005) Germinal center exclusion of autoreactive $B$ cells is defective in human systemic lupus erythematosus. J. Clin. Invest. 115(11):3205-16.

43. Chang MK, et al. (2004) Apoptotic cells with oxidation-specific epitopes are immunogenic and proinflammatory. J. Exp. Med. 200(11):1359-70.

44. Martin SJ, et al. (1995) Early redistribution of plasma membrane phosphatidylserine is a general feature of apoptosis regardless of the initiating stimulus: inhibition by overexpression of Bcl-2 and Abl. J. Exp. Med. 182(5):1545-56.

45. Berger NA, Petzold SJ. (1985) Identification of minimal size requirements of DNA for activation of poly(ADP-ribose) polymerase. Biochemistry. 24(16):4352-55.

46. Deveraux QL, et al. (1999) Cleavage of human inhibitor of apoptosis protein XIAP results in fragments with distinct specificities for caspases. EMBO J. 18(19):5242-51.

47. Yamada A, Cohen PL, Winfield JB. (1985) Subset specificity of antilymphocyte antibodies in systemic lupus erythematosus. Preferential reactivity with cells bearing the $\mathrm{T} 4$ and autologous erythrocyte receptor phenotypes. Arthritis Rheum. 28(3):262-70.

48. Dorsett B, Cronin W, Chuma V, Ioachim HL. (1985) Anti-lymphocyte antibodies in patients with the acquired immune deficiency syndrome. Am. J. Med. 78(4):621-26.

49. Minota S, Winfield JB. (1987) Identification of three major target molecules of IgM antilymphocyte autoantibodies in systemic lupus erythematosus. J. Immunol. 139(11):3644-51.

50. Bhat NM, et al. (1997) Rapid cytotoxicity of human B lymphocytes induced by VH4-34 (VH4.21) gene-encoded monoclonal antibodies, II. Clin. Exp. Immunol.108(1):151-59.

51. Baliakas P, et al. (2014) Clinical effect of stereotyped B-cell receptor immunoglobulins in chronic lymphocytic leukaemia: a retrospective multicentre study. Lancet Haematol. 1(2):e74-e84.

Cite this article as: Catera $\mathrm{R}$, et al. (2017) Binding of CLL subset 4 B cell receptor immunoglobulins to viable human memory B lymphocytes requires a distinctive IGKV somatic mutation. Mol. Med. 23:1-12. 